\title{
Sintomas auditivos em usuários de estéreos pessoais
}

\section{Hearing symptoms personal stereos}

\author{
Tiara Santos da Luz', Ana Lúcia Vieira de Freitas Borja².
}

1) Graduação em Fonoaudiologia. Fonoaudióloga.

2) Mestre em Medicina e Saúde Humana pela Fundação Bahiana para o Desenvolvimento das Ciências, FBDC, Brasil. Docente da Universidade Federal da Bahia.

Instituição: Universidade Federal da Bahia - Instituto da Ciência da Saúde. Salvador / BA - Brasil.

Endereço para correspondência: Tiara Santos da Luz - Rua Augusto Viana s/n Bairro: Vale do Canela - Salvador / BA - Brasil - CEP: $40110-060$ - Telefone: (+55 71) 8628-7013 - E-mail: tiaraluz87@ hotmail.com

Artigo recebido em 15 de junho de 2011. Artigo aprovado em 14 de novembro de 2011

\section{RESUMO}

Introdução: Práticos e portáteis os estéreos pessoais se tornaram acessórios quase indispensáveis no dia a dia. Estudos revelam que os tocadores de música portáteis podem causar danos auditivos a longo prazo para quem ouve música em alto volume por um tempo prolongado.

Objetivo: verificar a prevalência de sintomas auditivos em usuários de tocadores amplificados e conhecer os seus hábitos de uso

Método: Estudo prospectivo observacional de corte transversal realizado em três instituições de ensino da cidade de Salvador- BA, sendo duas de rede pública e uma da rede privada. Responderam ao questionário 400 estudantes, de ambos os sexos, entre 14 e 30 anos que referiram o hábito de utilizar estéreos pessoais.

Resultados: Os sintomas mais prevalentes foram hiperacusia $(43,5 \%)$, plenitude auricular $(30,5 \%)$ e zumbido $(27,5)$, sendo que o zumbido é o sintoma mais presente na população mais jovem. Quanto aos hábitos diários: 62,3\% usam frequentemente, $57 \%$ em intensidades elevadas, $34 \%$ em períodos prolongados. Verificou-se uma relação inversa entre tempo de exposição e a faixa de idade $(\mathrm{p}=0,000)$ e direta com a prevalência do zumbido.

Conclusão: Apesar de admitirem ter conhecimento sobre os danos que a exposição a som de alta intensidade pode causar a audição, os hábitos diários dos jovens evidenciam o uso inadequado dos estéreos portáteis caracterizados por longos períodos de exposição, intensidades elevadas, uso frequente e preferência pelos fones de inserção. A alta prevalência de sintomas após o uso sugere um risco maior para a audição desses jovens.

Palavras-chave: audição, sintomas, hábitos.

\section{SUMMARY}

Introduction: Practical and portable the personal stereos if had become almost indispensable accessories in the day the day. Studies disclose that the portable players of music can cause auditory damages in the long run for who hear music in high volume for a drawn out time.

Objective: to verify the prevalence of auditory symptoms in users of amplified players and to know its habits of use

Method: Observational prospective study of transversal cut carried through in three institutions of education of the city of Salvador BA, being two of public net and one of the private net. 400 students had answered to the questionnaire, of both the sex, between 14 and 30 years that had related the habit to use personal stereos.

Results: The symptoms most prevalent had been hyperacusis (43.5\%), auricular fullness (30.5\%) and humming (27.5), being that the humming is the symptom most present in the population youngest. How much to the daily habits: $62.3 \%$ frequent use, $57 \%$ in raised intensities, $34 \%$ in drawn out periods. An inverse relation between exposition time was verified and the band of age $(p=0,000)$ and direct with the prevalence of the humming. Conclusion: Although to admit to have knowledge on the damages that the exposition the sound of high intensity can cause the hearing, the daily habits of the young evidence the inadequate use of the portable stereos characterized by long periods of exposition, raised intensities, frequent use and preference for the insertion phones. The high prevalence of symptoms after the use suggests a bigger risk for the hearing of these young.

Keyword: hearing, symptoms, habits. 


\section{INTRODUÇÃO}

Os aparelhos individuais com fone de ouvido revolucionaram a forma de ouvir música. Por serem práticos e portáteis se tornaram acessórios quase indispensáveis no dia a dia. Esse hábito moderno tem mobilizado os pesquisadores para estudar o impacto negativo do uso inadequado desses equipamentos sobre a audição. Um estudo recente revela que os tocadores de música portáteis podem causar danos auditivos a longo prazo para quem ouve música em alto volume por um tempo prolongado (1).

A audição é uma das principais vias pelas qual o ser humano interage com o meio, sendo um dos sentidos mais importantes, além disso, tem uma função bastante complexa e primordial na comunicação humana e preservação da espécie. É por meio dela que ouvimos e identificamos todos os sons do ambiente. Lesões nas estruturas sensoriais da audição provocam prejuízos na detecção, localização e discriminação dos sons.

O crescimento da urbanização e o avanço da tecnologia favoreceram a elevação dos níveis de ruído nas ruas, no trabalho e no lazer, acarretando prejuízo ao bem estar físico e mental, bem como a audição dos indivíduos (2). Autores ratificam essa afirmação, e acrescentam que o ruído não afeta apenas a audição, mas também podem produzir sintomas como aumento da pressão arterial, distúrbios gastrointestinais, insônia e irritabilidade (3). Atualmente, o ruído ambiental tem sido considerado um problema de saúde pública por fazer parte da maioria das atividades cotidianas do ser humano (4).

Os jovens, em sua maioria, adolescentes, habitualmente estão expostos a música amplificada de alta intensidade, especialmente nas suas atividades de lazer (5). A música, em geral, é um som agradável que proporciona sensação prazerosa, entretanto, pode se tornar uma fonte de poluição sonora dependendo da maneira e do nível sonoro que é utilizado (4).

Observa-se que adolescentes na faixa etária de 12 a 18 anos, aumentaram muito o uso de equipamentos estéreos pessoais (EP), muitas vezes, usados de maneira inadequada, por muitas horas e em intensidades muito elevadas. Há dois motivos preocupantes que fazem com que esses modernos equipamentos sejam prejudiciais para usuário: o primeiro é a grande capacidade de memória e alta durabilidade da bateria, o que favorece seu uso em jornadas extensas. E o segundo é o design dos fones, cuja tendência atual é o uso dos fones de inserção que são capazes de concentrar toda a energia sonora produzida dentro do conduto auditivo externo, sendo considerados, por esta característica, os mais prejudiciais (6).
Outro aspecto preocupante é o nível de intensidade com que esses equipamentos são utilizados, permitindo que pessoas a um metro de distância escutem a música que esta sendo ouvida pelo usuário através dos fones. Estudos demonstraram que os níveis de pressão sonora desses equipamentos podem atingir $120 \mathrm{~dB}$, intensidade suficiente para provocar lesão auditiva $(7,8$, 9).

O grande número de estudos acerca deste tema evidencia a preocupação de que o uso desses equipamentos de modo abusivo e incorreto possa causar danos irreversíveis à audição $(2,6)$.

Desta maneira, considerando a relevância do tema, o objetivo deste trabalho foi verificar a prevalência de sintomas referidos por usuários de tocadores de música digital amplificada e conhecer os seus hábitos em relação ao uso desses equipamentos.

\section{MÉTODO}

\section{Desenho do estudo e população}

Trata-se de um estudo observacional tipo corte transversal, conduzido com dados primários, realizado na cidade de Salvador/BA no período de setembro a novembro de 2010. Foi desenvolvido em três instituições, uma do ensino médio e duas do ensino superior, sendo duas de rede pública e uma de rede privada.

Participaram da pesquisa 400 estudantes, de ambos os sexos, com faixa etária entre 14 e 30 anos que referiram o hábito de utilizar estéreos pessoais. A amostra foi composta por estudantes do ensino médio e do ensino superior em faculdades públicas e privadas, independente do curso.

\section{Coleta de dados e Aspectos Éticos}

Os dados foram obtidos através de um questionário estruturado (Apêndice A) sobre os hábitos diários de uso dos estéreos pessoais, sintomas auditivos após uso desses equipamentos e o grau de conscientização acerca dos possíveis riscos que estes equipamentos podem causar a audição. Também foi utilizada uma escala analógica visual, a fim de estimar a faixa de volume habitualmente utilizada pelos usuários, a mesma consiste de uma reta de $10 \mathrm{~cm}$ desprovida de números, na qual há apenas indicações na extremidade à esquerda de volume mínimo, que corresponde a $0 \mathrm{~cm}$ e à direita volume máximo que corresponde $10 \mathrm{~cm}$. 


\section{Apêndice A. Questionário.}

Instituição de ensino:

Escolaridade: ( ) Fundamental ( ) Médio ( ) Superior

Idade:

I. Você faz uso de estéreos pessoais (Mp3, Mp4, Ipods)?

( ) $\operatorname{Sim}($ ) Não

2. Comque frequência você costuma usar seu estéreos pessoais?

( ) Sempre ( ) Ás vezes ( ) Raramente

3. Quantas horas por dia você usa seu estéreos pessoais?

( ) 30 min ( ) Ih ( ) Mais de $2 \mathrm{~h} \mathrm{(} \mathrm{)} \mathrm{Mais} \mathrm{de} 4 \mathrm{~h}$

4. Na escala abaixo marque em qual posição do volume você considera usar habitualmente seu estéreo pessoal, sendo 0 o volume mínimo e 10 o volume máximo.

0

5. Você sabe qual o volume máximo de seu equipamento (Mp3, Mp4, Ipod)?

( ) $\operatorname{Sim}($ ) Não

6. Qual tipo de fone de ouvido você costuma usa seu estéreo pessoal?

( ) Inserção (coloca dentro do canal auditivo) ( ) Circum aural (fica sobre a orelha)

7. Emqual ambiente você costuma usar seu estéreo pessoal?

( ) Ambientes ruidosos ( ) Ambientes silenciosos ( ) Em ambos

8. Você costuma dormir ouvindo música comseu estéreo pessoal?

( ) $\operatorname{Sim}($ ) Não

9. Qual ogênero musical você costuma ouvir emseu estéreo? (pode marcar mais de uma alternativa)

( ) Rock ( ) Pagode ( ) Sertanejo ( ) Jazz ( ) MPB ( ) Samba

10. Vocêacha que ouve bem?

( ) $\operatorname{Sim}$ ( ) Não

I I. Após uso do estéreo (Mp3, Mp4, Ipod), já percebeu algum desses sintomas:

( ) Zumbido ( ) Dor ( )Tontura ( ) Sensação de ouvido tapado

( ) Diminuição da audição ( ) Outros

12. Tem dificuldade em perceber ou discriminar sons, ou em compreender afala, emambientes ruidosos?

( ) $\operatorname{Sim}($ ) Não

13. Quando ouve sons fortes ou está em ambientes muito ruidosos sente desconforto nos ouvidos?

( ) $\operatorname{Sim}($ ) Não

14. Você acredita que o uso de estéreo pessoal em volume máximo pode causar perda auditiva?

( ) $\operatorname{Sim}$ ( ) Não

15. Já obteve alguma informação sobre os efeitos nocivos que o ruído pode trazer para saúde?

( ) Não ( ) Sim.

16. Caso tenha respondido simà pergunta anterior, porque meio teve acesso a essa informação?

( ) Escola ( ) TV, rádio ( ) Outdoor ( ) Mídia escrita (jornal, revista)

( ) Internet ( ) Profissional de saúde

Para posterior análise da escala, foram feitas as seguintes considerações:

\section{Quadro I. Correspondência da escala analógica visual.}

0 10

Escala emcentímetros $(\mathrm{cm})$ Faixa de volume

$0-4,5 \quad$ Volume adequado/Baixo Risco

4,6-7,5 Volume moderado/Risco Moderado

7,6- 10 Volume alto/Alto Risco

Previamente à aplicação dos questionários as instituições que participaram da pesquisa assinaram um termo de consentimento autorizando a aplicação dos questionários nas suas dependências, bem como, todos os partici- pantes também assinaram um termo de consentimento livre e esclarecido.

Este estudo foi submetido ao Comitê de Ética em pesquisa da Faculdade de Tecnologia e Ciência (FTC), tendo sido aprovado, sem restrições através do parecer no 2037.

\section{Análise de Dados}

Os dados foram digitados no programa EpiData Entry 3.1 (CDC, Atlanta, GA) e analisados, quantitativamente, no SSPS versão 15 . Os dados foram tabulados e apresentados em forma de Tabela e Gráficos. 
Para variáveis categóricas foram estimadas frequências simples e comparadas com o teste qui - quadrado de Pearson. Enquanto para variáveis contínuas foram estimadas médias e medianas. Foram considerados como resultados estatisticamente significantes aqueles que apresentam p-VALOR $<$ que 0,05 .

\section{RESULTADOS}

A Tabela 1 mostra os dados sócio-demográficos da população. Participaram da pesquisa 200 alunos do ensino médio e 200 do ensino superior destes 300 pertenciam à instituição pública e 100 de privada. A faixa etária variou entre 14 e 30 anos, entretanto 91,6\% tinham idade até 25 anos, 64,8\% eram do sexo feminino.

A Tabela 2 evidencia os resultados quanto aos hábitos diários de exposição à música eletronicamente amplificada em aparelhos individuais. Quantoà frequência de uso observa-se que $62,3 \%$ dos participantes referiram usar frequentemente, $31,7 \%$ às vezes, $6 \%$ raramente.

No que diz respeito à faixa de volume de uso habitual, 57\% dos indivíduos afirmam ouvir em volume alto, 33\% em volume moderado, e apenas 10\% em volume baixo. Vale ressaltar que 50,8\% relataram ter conhecimento do volume máximo de saída do seu equipamento.

Verificou-se ainda, que 34\% dos jovens costumam ouvir música por um período que varia de 2 até 6 h por dia. Observou-se uma correlação inversa entre o tempo de exposição e a faixa de idade $(\mathrm{p}=, 000)$. Quanto a o tipo de fone $75,5 \%$ afirmaram utilizar fone de inserção, 20,8\% fone circum aural e 3,3\% utilizam ambos os fones.

Os gêneros musicais mais presentes na preferência dos participantes são a MPB com 63\% seguido de rock $(48,3 \%)$ e pagode $(41,5 \%)$.

Tabela I. Caracterização da amostra.

\begin{tabular}{llcc}
\hline Variáveis & & $N(400)$ & $\%$ \\
\hline Idade(anos) & $\mid 4$ a 18 & 205 & $51,3 \%$ \\
& 19 a 25 & 161 & $40,3 \%$ \\
Sexo & 26 a 30 & 34 & $8,4 \%$ \\
& Feminino & 259 & $64,8 \%$ \\
Instituição & Masculino & $|4|$ & $35,2 \%$ \\
& Pública & 300 & $75,0 \%$ \\
Escolaridade & Privada & 100 & $25,0 \%$ \\
& NívelMédio & 200 & $50,0 \%$ \\
& NívelSuperior & 200 & $50,0 \%$ \\
\hline
\end{tabular}

Tabela 2. Hábitos relacionados ao uso de estéreos pessoais.

\begin{tabular}{|c|c|c|c|}
\hline Variáveis & & $N(400)$ & $\%$ \\
\hline Tempo de exposição/dia & $\begin{array}{l}\text { Até } 1 / 2 \mathrm{~h} \min \\
\text { Entre } 1 / 2 \mathrm{he} 2 \mathrm{~h} \\
\text { Entre } 2 \mathrm{~h} \text { de } 6 \mathrm{~h}\end{array}$ & $\begin{array}{l}55 \\
117 \\
136\end{array}$ & $\begin{array}{r}13,80 \% \\
29.3 \% \\
34,00 \%\end{array}$ \\
\hline Faixa de volume habitual & $\begin{array}{l}\text { Volume baixo } \\
\text { Volume moderado } \\
\text { Volume alto }\end{array}$ & $\begin{array}{l}40 \\
132 \\
228\end{array}$ & $\begin{array}{l}10,00 \% \\
33,00 \% \\
57,00 \%\end{array}$ \\
\hline Sabe volume máximo? & $\begin{array}{l}\text { Sim } \\
\text { Não }\end{array}$ & $\begin{array}{l}203 \\
195\end{array}$ & $\begin{array}{l}50,80 \% \\
48,80 \%\end{array}$ \\
\hline Tipo de fone & $\begin{array}{l}\text { Inserção } \\
\text { Circumaural } \\
\text { Ambos }\end{array}$ & $\begin{array}{c}302 \\
83 \\
13\end{array}$ & $\begin{array}{r}75,50 \% \\
20,80 \% \\
3,30 \%\end{array}$ \\
\hline Ambiente de uso & $\begin{array}{l}\text { Ruidoso } \\
\text { Silencioso } \\
\text { Ambos }\end{array}$ & $\begin{array}{c}55 \\
60 \\
285\end{array}$ & $\begin{array}{l}13,70 \% \\
15,00 \% \\
71,30 \%\end{array}$ \\
\hline Dorme ouvindo música? & $\begin{array}{l}\text { Sim } \\
\text { Não }\end{array}$ & $\begin{array}{l}187 \\
209\end{array}$ & $\begin{array}{l}46,80 \% \\
52,30 \%\end{array}$ \\
\hline Gênero musical & $\begin{array}{l}\text { MPB } \\
\text { Rock } \\
\text { Pagode } \\
\text { Samba } \\
\text { Sertanejo } \\
\text { Jazz } \\
\text { Outros }\end{array}$ & $\begin{array}{l}252 \\
193 \\
166 \\
154 \\
91 \\
85 \\
18\end{array}$ & $\begin{array}{r}63,00 \% \\
48,30 \% \\
41,50 \% \\
38,50 \% \\
22,80 \% \\
21,30 \% \\
4,50 \%\end{array}$ \\
\hline
\end{tabular}


A maioria dos entrevistados $(71,3 \%)$ costuma utilizar o player portátil tanto em ambientes ruidosos quanto silenciosos, porém, $13,7 \%$ utilizam somente em ambiente ruidoso; $46,8 \%$ referem o hábito de dormir ouvindo música nos estéreos pessoais.

Quanto à presença de sintomas após exposição à música eletronicamente amplificada (Tabela 3), 67,2\% dos entrevistados já apresentaram pelo menos um sintoma, sendo que $18,7 \%$ referiram mais de um sintoma.

Os sintomas mais prevalentes foram plenitude auricular $(30,5 \%)$ seguido de zumbido $(27,5 \%)$, otalgia $(12,8 \%)$, hipoacusia $(11,5 \%)$, tontura $(4,8 \%)$ e outros $(4,0 \%)$.34\% não referiram sintomas após o uso. Verificouse que quanto maior a idade menor a presença de sintomas após o uso e quanto menor a faixa etária maior é a prevalência do zumbido nesta população. Também observa-se uma relação direta entre o tempo de exposição e o aumento da prevalência do zumbido. Vale ressaltar, que o uso em intensidades elevadas guarda uma relação direta com a quantidade de sintomas referidos.

Como queixa permanente $43,5 \%$ dos participantes afirmaram apresentar desconforto a sons intensos e dificuldade em perceber sons e compreender fala em ambientes ruidosos (36,5\%). Sendo que 15,8\% referem não ouvir bem.

Foi evidenciado que quanto maior o tempo de exposição maior a prevalência de queixa de zumbido.

No que diz respeito ao grau de conscientização em relação aos possíveis riscos que o uso de estéreos pessoais podem causar a audição, $91,5 \%$ acreditam que estes podem causar perda auditiva e $75,5 \%$ já obtiveram alguma informação sobre os efeitos nocivos que o ruído pode trazer para saúde. Os meios de maior acesso a essas informações foram TV/rádio (40,8\%), internet (29,8\%), profissionais de saúde(26\%), mídia escrita $(24,8 \%)$ e escola $(23,8 \%)$.

\section{DISCUSSÃO}

Por serem práticos, portáteis e estarem na moda os equipamentos estéreos pessoais se tornaram acessórios quase indispensáveis no dia-a-dia, principalmente entre os jovens. Este hábito de consumo moderno tem gerado grandes discussões e pesquisas no meio científico acerca dos prejuízos que podem causarà audição desses indivíduos.

Chama a atenção o fato de que o uso desses equipamentos, embora mais comum entre adolescentes, está presente hoje em todas as faixas de idade. Esse hábito está cada vez mais incorporado à rotina das pessoas e o uso inadequado torna-se num risco à saúde auditiva.
Tabela 3. Sintomas e queixas relacionadas à audição.

\begin{tabular}{llcr}
\hline Variáveis & & $\mathrm{N}(400)$ & $\%$ \\
\hline Quantidades desintomas & I & 194 & $48,50 \%$ \\
& + de I & 75 & $18,70 \%$ \\
Sintomas após uso de & P.Auricular & 122 & $30,50 \%$ \\
estéreo pessoal & Zumbido & 110 & $27,50 \%$ \\
& Otalgia & 51 & $12,80 \%$ \\
& Hipoacusia & 46 & $11,50 \%$ \\
& Tontura & 19 & $4,8 \%$ \\
& Outros & 16 & $4,0 \%$ \\
Dificuldade emperceber & Sim & 146 & $36,50 \%$ \\
e compreenderafala & Não & 254 & $63,50 \%$ \\
emambientes ruidosos & & & \\
Desconforto a sons & Sim & 174 & $43,50 \%$ \\
intensos & Não & 225 & $56,30 \%$ \\
Achaque ouve bem & Sim & 334 & $83,50 \%$ \\
& Não & 63 & $15,80 \%$ \\
\hline
\end{tabular}

Apesar da dimensão reduzida, esses equipamentos estão cada vez mais potentes, com grande capacidade de memória e alta durabilidade da bateria favorecendo seu uso em jornadas extensas (6). Além disso, estudos mostram que os níveis de pressão sonora desses equipamentos podem atingir $120 \mathrm{~dB}$, intensidade de risco potencial ao ouvido humano $(7,8,9)$.

Constatou-se que $62,3 \%$ da população estudada têm o hábito de usar frequentemente estéreos pessoais por longos períodos de exposição e em intensidades elevadas, estes achados corroboram outros estudos com esta população $(2,8,9,10,11,12)$.

Os riscos à saúde auditiva ficam evidentes quando observamos os hábitos de uso e a correlação com os sintomas auditivos. Neste sentido, há uma correlação direta entre o tempo de exposição e a presença de sintomas auditivos $(p=0,004)$ destacando-se o zumbido como o sintoma cuja correção tem maior aderência estatística. Relação estatisticamente significante também foi encontrada entre o tempo de exposição e a faixa de idade $(\mathrm{p}=, 0000)$.

A utilização destes equipamentos portáteis dá-se em diversos ambientes ruidosos como no trânsito, escola, faculdade, rua, academia de ginástica etc. Em geral, nesses locais, o nível de ruído é elevado o que faz com que os usuários aumentem o volume para encobrir sons externos $(11,12)$. Observou-se neste estudo que $81,5 \%$ dos jovens costumam utilizar estéreos pessoais em ambientes ruidosos.

Outro aspecto a ser considerado é o design dos fones de ouvido cujo modelo pode potencializar a capacidade sonora desses equipamentos. Os fones de inserção 
por serem discretos, mais estéticos e por possuírem praticidade superior aos volumosos fones externos que recobrem a orelha (circum-aural), são atualmente os mais populares (6). Nesta população observou-se que 75,5\% têm preferência pelos fones de inserção, concordando com outros autores $(4,6,9)$. A posição deste tipo de fone, inserido no conduto auditivo externo, favorece a potencialização da intensidade visto que toda a pressão sonora é conduzida para orelha média. Para GARSTECKI (2006), professor de desordens e ciências de comunicação da universidade Northwestem (EUA), o problema se agrava quando estes pequenos fones não se encaixam perfeitamente nos ouvidos e permitem a entrada de sons externos, o que faz com que o usuário aumente mais o volume do player para abafar o barulho externo (13).

Um número significativo de jovens 67,2\%, afirmaram ter apresentado pelo menos um dos sintomas após exposição à música eletronicamente amplificada. Os sintomas auditivos mais referidos pelos usuários de estéreos pessoais foram plenitude auricular, seguido de zumbido, estes achados foram similares aos encontrados em outros estudos (2, 5, 8, 9, 14, 15). Alguns autores acreditam que o zumbido, a sensação de ouvido tapado e diminuição da audição após exposição excessiva à música amplificada, podem ser os primeiros sinais de perda auditiva induzida pela música $(9,14)$.

É consenso na literatura a presença de mudanças temporárias do limiar (TTS) após exposição a ruídos de alta intensidade (4, 9, 14, 15, 16, 17). Todavia, mudanças temporárias decorrentes de superexposição ao ruído podem resultar em mudanças permanentes do limiar $(3,18)$. Os principais fatores para o desenvolvimento de perda auditiva são o tempo de exposição, intensidade sonora e a suscetibilidade individual $(3,15,18)$.

Por outrolado, perda auditiva para alguns autores não é a única lesão resultante da exposição a doses de ruídos superpostos. Esses indivíduos podem apresentar queixas como zumbido crônico, recrutamento e hipersensibilidade ao som (hiperacusia) (19). Este estudo constatou que grande parte dos participantes (43,5\%) referiu desconforto a sons intensos, concordando com outros estudos (3). Hiperacusia ou hipersensibilidade aos sons é o constante incomodo a sons de intensidade fraca, moderada ou forte, independente da situação ou ambiente $(3,19)$.

Vale ressaltar, que lesões do órgão de corti, hiperacusia, zumbido, entre outras alterações podem impedir ou dificultar a utilização plena das habilidades auditivas, prejudicando a qualidade de vida dos indivíduos (19). Um número significativo dos respondentes 36,5\% referiu apresentar dificuldade em perceber sons e compreender fala em ambientes ruidosos, corroborando pesquisas similares $(2,4,8,10)$.
O impacto negativo do uso inadequado desses equipamentos sobre a audição, sem dúvida pertence ao campo da saúde pública (1). Os equipamentos portáteis individuais estão chegando cada vez mais cedo às mãos e ouvidos dos jovens, torna-se evidente a necessidade da implantação precoce de ações educativas desde o ensino fundamental. Neste estudo observou-se uma correlação inversa entre o tempo de exposição e a faixa de idade, ou seja, os mais jovens têm uma propensão a utilizar o player portátil em maiores intensidades $(p=, 000)$.

A maioria dos sujeitos entrevistados (91,5\%) acredita que o uso de estéreos pessoais em volume máximo pode causar perda auditiva e 75,5\% já obtiveram alguma informação sobre os efeitos nocivos que o ruído pode trazer para saúde, estes achados estão de acordo com outros estudos similares $(5,8)$. Contrariamente, em num estudo realizado em São Paulo 85\% dos jovens acreditam que o uso desses equipamentos em intensidade elevada não causa perda auditiva (6).

Apesar do amplo percentual de jovens que já tiveram acesso a informações sobre os efeitos nocivos do ruído sobre a saúde, os a chados a qui descritos demonstram que um grande contingente permanece com hábitos de uso inadequados desses equipamentos. Vários autores discutem a falta de ações de proteção e prevenção das alterações auditivas ocasionadas especificamente por exposição à música amplificada $(2,4)$.

A escola é o meio de informação menos citado pelos participantes (23,8\%) e o mais citado a TV/Rádio (40,8\%). Outros meios citados foram a internet (29,8\%), profissionais de saúde (26,0\%) e a mídia escrita (23,8\%). Estes números sugerem uma participação insignificante da escola frente ao papel que desempenha na formação dos jovens.

\section{ConClusão}

Os sintomas auditivos mais prevalentes nos usuários de equipamentos portáteis individuais foram desconforto a sons intensos, dificuldade em perceber sons e compreender fala em ambientes ruidosos, plenitude auricular, zumbido, otalgia e hipoacusia. Quanto maior a idade menor a presença de sintomas após o uso e quanto menor a faixa etária maior é a prevalência do zumbido nesta população

Os hábitos diários de exposição evidenciam o uso inadequado desses equipamentos caracterizados por longos períodos de exposição, intensidades elevadas, uso frequente e preferência pelos fones de inserção.

Apesar de quase a totalidade $(91,5 \%)$ dos jovens acreditarem que o uso de estéreos pessoais em volume 
máximo possa causar perda auditiva e referirem informações prévias sobre os efeitos nocivos do ruído sobre a saúde, um grande contingente permanece com hábitos de uso inadequados desses equipamentos.

A escola figura como o meio de informação que menos contribuiu para a formação do conhecimento acerca dos efeitos nocivos do ruído. Sugere-se uma participação mais ativa da escola frente ao papel que desempenha na formação dos jovens, desenvolvimento de ações educacionais sobre os riscos que a exposição inadequada aos ruídos pode causar a saúde.

\section{REFERÊNCIAS BIBLIOGRÁFICAS}

1. Tarantino M. Cuidado: o som alto do seu tocador portátil pode provocar perda auditiva. Revista eletrônica Medicina e Bem Estar capturada, novembro 2006. Disponível em: http://www.terra.com.br/istoe/1932/medicina/ 1932_abaixe_o_volume.htm Acesso em 15.11.09

2. Bahia CS, Borja ALV. Sintomas auditivos referidos pelos usuários de player portátil. In: XVIII Congresso Brasileiro de Fonoaudiologia. Anais; 2009. Salvador (BA); 21 a 24 de outubro. p. 2141.

3. Morata TC, Santos UP. Efeitos do ruído na audição. In: Santos UP. Ruído: riscos e prevenção. $1^{1}$ Ed. São Paulo: Hucitec; 1999, pp. 43-53.

4. Ventura CT, Fiorini AC. Música amplificada: uma revisão sobre seus efeitos na saúde. In: XVIII Congresso Brasileiro de Fonoaudiologia. Anais; 2009. Salvador(BA); 21 a 24 de outubro. p. 2698.

5. Borja ALV, Souza BF, Ramos MM, Araújo RPC. O que os jovens adolescentessabemsobre as perdas auditivasinduzidas excesso de ruído? Rev Ciênc Méd e Biol. 2002, 1(1):86-98.

6. Russo ICP, First D, Abud NCD. El uso del estéreo personal: conocimiento y la concienciade los adolescentes. Asha. 2009, 1:22-37.

7. Momensonh-Santos TM, Freitas TVD, Molinaro MCG, Lamas C, Bueno C, Nogueira M. O uso dos equipamentos de som pessoais e a presença de zumbido. In: XVIII Congresso Brasileiro de Fonoaudiologia. Anais; 2009. Salvador (BA); 21 a 24 de outubro. p. 2201.

8. Nascimento DP, Santos APB, Yonezaki C. Efeitos do uso excessivo de equipamentos eletroportáteis em altos níveis sonoros na audição. In: XVII Congresso Brasileiro de Fonoaudiologia. Anais; 2008. Campos Jordão (SP); 24 a 27 de setembro. p. 1149.
9. Gonçalves VSb, Lacerda JMV, Brito LKB, Oliveira NCM. Estudo dos hábitos auditivos em estudantes de escola privadas na cidade de João Pessoa. In: XVIII Congresso Brasileiro de Fonoaudiologia. Anais; 2009. Salvador (BA); 21 a 24 de outubro. p. 2417.

10. Jorge Jr. JJ, ALegre ACM, Greco MC, Angelini MCA, Barros PM. Hábitos e limiares auditivos de jovens em relação à música eletronicamente amplificada através de equipamentos com fone de ouvido. Rev Bras de Otorrinolaringol. 1996, 62(6):424-34.

11. Momensonh-Santos TM, Lopes MKD. Uso de sistemas de som estéreos pessoais em um grupo de trabalhadores. In:XVIICongresso Brasileiro de Fonoaudiologia. Anais; 2008. Campos Jordão (SP); 24 a 27 de setembro. p. 173.

12. Garstecki D. Fones de ouvido IPods podem danificar audição. Plantão INFO, janeiro. 2006. Disponível em: http:/ /info.abril.com.br/aberto/infonews/012006/020120066.shl. Acesso em 20 de out. 2010.

13. Andrade AIA, Russo ICP, Lima MLLT, Oliveira LCS. Avaliação auditiva em músicos de frevo e maracatu. Rev Bras de Otorrinolaringol, 2002, 68(5):714-720.

14. Jorge JrJJ, Alegre ACM. A audição dos jovens e sua relação com hábitos de exposição à música eletronicamente amplificada. Introdução ao tema e uma revisão bibliográfica. Rev Bras de Otorrinolaringol. 1995, 61(1):7-13.

15. Russo ICP, Momensohn TM, Busgaib BB, Osterne FGJ. Um estudo comparativo sobre os efeitos da exposição à música em músicos de trio elétrico. Rev Bras de Otorrinolaringol. 1995, 61(6):477-484.

16. Silveira JAM, Brandão ALA, Rossi JD, Ferreira LLA, Name MAM, Estefan P, Gonçalez F. Avaliação da alteração auditiva provocada pelo uso de walkman, por meio da audiometria tonal e das emissões otoacústicas (produto de distorção): estudo da 40 orelhas. Rev Bras de Otorrinolaringol. 2001, 67(5):650-654.

17. Russo ICP. Ruído, seus efeitos e medidas preventivas. In: ___ (Org). Acústica e Psicoacústica aplicada à fonoaudiologia. 1를. São Paulo: Lovise;1999, pp. 157- 169.

18. Santos-Momensohn TM, Duran A. Risco de perda auditiva por uso de discman: estudo sobre os níveis de pressãosonora medidos em sistema de CD player. Rev Distúrbio da Comunicação. 1995, 61(3):7-13.

19. Tochetto TM, Gonçalves MS, Gambini C. Hiperacusia em músicos da banda militar. Rev Soc Bras Fonoaudiol. 2007, 12(4):298-303. 